\title{
On the structure of quasi-modular spaces
}

\author{
by
}

TETSUYA SHIMOGAKI (Sapporo)

\$1. Introduction. Let $R$ be a universally antinuous semi-ordered linear space (1) (i. e. a conditionally complete vector-lattice in Birkhoff's sense [1]) and $\varrho$ be a functional satisfying the following conditions:

(p.1) $0 \leqslant \varrho(a)=\varrho(-a) \leqslant+\infty$ for all $a \in R$;

(р.2) $\varrho(a+b)=\varrho(a)+\varrho(b)$ if $a \perp b\left({ }^{2}\right)$;

(ค.3) for any orthogonal system $\left\{a_{\lambda}\right\}_{(\lambda \in \Lambda)}$ with $\sum_{\lambda \in A} \varrho\left(a_{\lambda}\right)<+\infty$, there exists $a_{0} \epsilon R$ such that $a_{0}=\sum_{i \in \Lambda} a_{\lambda}$ and $\varrho\left(a_{0}\right)=\sum_{\lambda \in \Lambda} \varrho\left(a_{\lambda}\right)$;

(९.4) $\varlimsup_{a \rightarrow 0} \varrho(\alpha a)<+\infty$ for all $a \in R$.

$R$ associated with $\varrho$ (denoted by $(R, \varrho)$ shortly) is called a quasimodular space and $\varrho$ is called a quasi-modular. The quasi-modular space was first defined in [3] and discussed in [3] and [4].

In [10] Nakano established the theory of modular spaces ( $\left.{ }^{3}\right)$, where a modular $m(a)(a \epsilon R)$ is a functional on $R$ satisfying (p.1), (p.2) and the additional conditions :

(i) $m(\xi a)$ is a convex function of real $\xi \geqslant 0$ which is not identically zero but finite in a neighbourhood of 0 (depending on a) in $[0,+\infty)$ for each $0 \neq a \in R$

(ii) $|a| \leqslant|b|$ implies $m(a) \leqslant m(b)$;

(iii) $0 \leqslant a_{\lambda} \uparrow_{\lambda \in \Lambda} a$ implies $m(a)=\sup _{\lambda \in \Lambda} m\left(a_{\lambda}\right)$.

A modular $m$ is called monotone complete, if $\sup _{\lambda \in \Lambda} m\left(a_{\lambda}\right)<+\infty$ and $0 \leqslant a_{\lambda} \uparrow_{\lambda \in \Lambda}$ imply $\bigcup_{\lambda \in A} a_{\lambda} \in R$ [10]. From above, it is easily seen that the

(1) This term is due to Nakano [10].

(2) $a \perp b, a, b \in R$, means that $|a| \cap|b|=0$ and for any set $M \subset R$ we denote by $M \perp$ the set $\{x: x \in R, x \perp y$ for all $y \in M\}$.

$\left({ }^{3}\right)$ Correctly it is called a modulared semi-ordered linear space in [10]. 
concept of a quasi-modular is a generalization of that of a monotone complete modular $\left({ }^{4}\right)$. On a modular space $(R, m)$ we can define a norm as

$$
\||a|\|=\inf _{m(\xi a) \leqslant 1} \frac{1}{|\xi|}\left({ }^{5}\right) \quad(a \in R),
$$

and hence we can consider $(R, m)$ as a normed space by this norm. If the modular $m$ is monotone complete, the norm is complete ([1.0], Theorem 38.6).

We denote by $\bar{R}$ the conjugate space of $R$, i. e. the totality of all universally continuous linear functionals $\left({ }^{6}\right)$ on $R$. $R$ is called semi-regular, if $\bar{R}$ is total $\left({ }^{7}\right)$ on $R . R$ is called non-atomic if $0 \neq a \in R$ can be decomposed into $a=b+c$ with $b \perp c$ and $b, c \neq 0$.

In the earlier paper [3] we proved the following theorem:

THEOREM A. If a quasi-modular space $(R, \varrho)$ is semi-regular and non-atomic, $R$ becomes a modular space $\left(R, m_{\rho}\right)$ with a (convex $\left.\left({ }^{8}\right)\right)$ perfect $\left({ }^{9}\right)$ modular $m_{e}$ constructed by $\varrho$.

A linear functional $\tilde{a}$ on $R$ is called bounded, if $\sup _{|x| \leqslant a}|\tilde{a}(x)|<+\infty$ for all $0 \leqslant a \in R$, and the totality of all bounded linear functionals on $R$ is called the associated space of $R$ and denoted by $\tilde{R}$. From the definition, it is clear that $\bar{R} \subseteq \tilde{R}$ and the equal sign does not hold in general.

The main aim of this paper is to improve Theorem A by replacing the assumption that $R$ is semi-regular by one that $\tilde{R}$ is total on $R$.

First we prove that, if $\tilde{R}$ is total on $R=(R, \varrho)$, there exists the normal manifolds $R_{r}$ and $R_{s}$ such that $R=R_{r} \oplus R_{s}\left({ }^{10}\right)$, where $R_{r}$ is semiregular and $R_{s}$ is $\varrho$-singular, i. e. $\varrho(a)=0$ or $+\infty$ for all $a \in R_{s}$ (Theorem 2.1). $R_{s}$ is also decomposed into $R_{s}=R_{\infty} \oplus \Re$, where $R_{\infty}$ has a strong unit and $\varrho(a)=0$ for all $a \in \Re$ (Theorems 2.2 and 2.3). The space $\Re$ is extremely pathological, if it exists, and intrests us by itself. $\S 3$ is devoted

(4) Recently the concept of a modular was also generalized and discussed by Musielak and Orlicz in [7] and [8]

$\left.{ }^{5}\right)$ It is called in [10] the second norm by $m$.

${ }^{(6)}$ A linear functional $f$ on $R$ is said to be universally continuous if $\inf \left|f\left(a_{\lambda}\right)\right|=0$ for any system of elements $\left\{a_{\lambda}\right\}_{\lambda \epsilon_{A}}$ with $a_{\lambda} \uparrow_{\lambda \in \Lambda} 0$.

(7) This means that if $\bar{a}(x)=0$ for all $\bar{a} \in \bar{R}$, then $x=0$.

${ }^{8}$. As is shown above, the essential difference between quasi-modulars and modulars is that the latter $m(\xi a)(a \in R)$ are convex functions for real coefficient $\xi$. To emphasize convexity of a modular, we use the term "convex modular" in place of "modular".

$\left.{ }^{9}\right)$ This means that every universally continuous linear functional is $\|\mid \cdot\| l-$ bounded.

( $\left.{ }^{10}\right)$ A manifold $M \subset R$ is called normal, if $\left(M^{\perp}\right)^{\perp}=M . R=M \oplus N$ means that $N=\left(M^{\perp}\right) \perp$ and each $a \in R$ is decomposed into $a=b+c, b \in M, c \in N$. to discuss $\Re$ and it is proved that every spectrum of $b$ by $a(a \neq 0, a, b \in \mathfrak{N})$ is a point spectrum.

In $\S 4$ we prove the main theorem (Theorem 4.1) which improves Theorem A, as is stated above, and some remarks are given in this direction. The improvement of Theorem A enables us to discuss $(R, \varrho)$ from the standpoint of linear topologies on $R$. In $\S 5$ we paraphrase the results obtained in $\S 4,[3]$ and [4] in terms of linear topologies $\mathfrak{T}$ on $(R, \varrho)$ and consequently show a generalization of a theorem due to Mazur and Orlicz ([6], 2.9). In fact we obtain that if a quasi-modular space $(R, \varrho)$ is topologized by a locally convex separated linear topology $\mathfrak{T}$ which is compatible and monotone complete, we may define a (convex) modular $m_{\mathrm{Q}}$ on $R$ such that Q-convergence (11) coincides with that of the norm induced by $m_{o}$. It is to be noted that as for the Mazur-Orlicz's Theorem there is the nice and faithful generalizations to abstract semi-ordered linear spaces and function spaces by Itô [2].

§ 2. Decomposition theorems. Let $(R, \varrho)$ be a quasi-modular space with a quasi-modular $\varrho$. We denote by $[p](p \in R)$ the projection operator defined by the set $\{p\}^{\perp . L}$, that is, $[p] a=\bigcup_{n=1}^{\infty}(n|p| \cap a)$ for all $0 \leqslant a \in R$ and we call $[p](p \in R)$ a projector by $p$.

From the definition of $\varrho$ we can easily prove that

and

$$
\varrho(0)=0, \quad \varrho(|a|)=\varrho(a)
$$

(2.2) $\varrho([p] a)=\sup _{\lambda \in A} \varrho\left(\left[p_{\lambda}\right] a\right)$ for each $a \in R$ and $\left[p_{\lambda}\right]_{\uparrow_{\lambda \in A}}[p]$.

In [4] we proved the following theorem:

THEOREM B. If $R$ is a quasi-modular space with a quasi-modular $\varrho$, the functional $\varrho^{\prime}$ defined by the formula

$$
\varrho^{\prime}(a)=\sup _{|x| \leqslant|a|} \varrho(x) \quad(a \in R)
$$

is also a quasi-modular on $R$ satisfying

(p.5) $|a| \leqslant|b|, a, b \in R$, implies $\varrho^{\prime}(a) \leqslant \varrho^{\prime}(b)$ ([4], Theorem 2.1).

In the argument below, it is convenient to utilize property (. .5$)$ which is not fulfilled by general quasi-modulars. So far as investigation of the structure of $(R, \varrho)$, however, we may assume that the quasi-modular $\varrho$ satisfies ( $\rho .5)$ by itself without loss of generality in virtue of Theorem B. Thus we let quasi-modulars $\varrho$ satisfy (p.5) in $\S 2$ and $§ 3\left({ }^{12}\right)$.

(11) A sequence of elements $\left\{a_{v}\right\} \underset{(v \geqslant 1)}{\subset} R$ is called $\varrho^{-c o n v e r g e n t}$ to $a$ if there is a fixed constant $K>0$ such that $\varlimsup_{p \rightarrow \infty} \varrho\left(\xi\left(a_{p}-a\right)\right) \leqslant K$ for all $\xi>0$ [3].

${ }^{(12)}$ The final result, however, comes to be free from (p.5) (Theorem 2.3). 
An element $a \in R$ is called $\varrho$-finite, if $\varrho(\alpha a)<+\infty$ for every real $\alpha \geqslant 0$. Let $F_{0}$ be the totality of all $\varrho$-finite elements. Now we have

LEMMA 1. $F_{0}$ is a semi-normal manifold (i.e. a linear manifold with the condition: $a \in F_{0}$ and $|a| \geqslant|b|, b \in R$ imply $\left.b \in F_{0}\right)$ of $R$.

Proof. The facts that $|a| \geqslant|b|$ and $a \in F_{0}$ imply $b \in F_{0}$ and that a $a \in F_{0}$ implies $2 a \in F_{0}$ are easily verified from the definition of $F_{0}$. Since $\varrho(a \cup b) \leqslant \varrho(a)+\varrho(b)$ and $|a+b| \leqslant 2(|a| \cup|b|)$ hold for any $a, b \in R$, we can conclude that $F_{0}$ is a linear manifold, q. e.d.

For any $\tilde{a} \epsilon \tilde{R}$ we denote by $\tilde{a}_{F_{0}}$ the functional $\tilde{a}$ restricted on $F_{0}$, i. $\theta ., \tilde{a}_{F_{0}}(x)=\tilde{a}(x)$ for all $x \in F_{0}$.

LEMMA 2. For any $\tilde{a} \in \tilde{R}, \tilde{a}_{F_{0}}$ is a continuous linear functional on $F_{0}$ (i. e. $\inf _{\nu \geqslant 1}\left|\tilde{a}_{F_{0}}\left(\left[p_{\nu}\right] a\right)\right|=0$ for any $\left[p_{\nu}\right] \downarrow_{\nu=1}^{\infty} 0$ and $\left.a \in F_{0}\left({ }^{13}\right)\right)$.

$\nu \geqslant 1$

Proof. Since $\tilde{a}$ is written as $\tilde{a}=\tilde{a}^{+}-\tilde{a}^{-}, \tilde{a}^{+}, \tilde{a}^{-} \geqslant 0\left({ }^{14}\right)$ and $\tilde{a}^{+} \perp \tilde{a}^{-}$ we may assume that $\tilde{a} \geqslant 0$ and $a \geqslant 0$ without loss of generality. Since $\left[p_{\mu}\right] \downarrow_{\mu=1}^{\infty} 0$ implies $\inf _{\mu \geqslant 1} \varrho\left(\left[p_{\mu}\right] \nu a\right)=0$ for easch $\nu \geqslant 1$ in virtue of $(p .2)$ and (2.2), we can find a subsequence $\left\{\left[p_{\mu \nu}\right]\right\}_{(v \geqslant 1)}$ of $\left\{\left[p_{\mu}\right]\right\}_{(\mu \geqslant 1)}$ such that $\varrho\left(\left[p_{\mu_{\nu}}\right] \nu a\right) \leqslant 1 / 2^{\nu}$ for all $\nu \geqslant 1$. We put $\left[q_{\nu}\right]=\left[p_{\mu_{\nu}}\right]-\left[p_{\mu_{\nu+1}}\right]$ and $b_{v}=\nu\left[q_{v}\right] a$ for all $\nu \geqslant 1$. Since $\left\{b_{v}\right\}_{(v \geqslant 1)}$ is an orthogonal sequence with $\sum_{v=1}^{\infty} \varrho\left(b_{\nu}\right)<+\infty$, there exists $0 \leqslant b_{0}=\sum_{\nu=1}^{\infty} b_{\nu} \in R$ by ( $\left.\rho .3\right)$. Then it follows from above

$$
\begin{aligned}
\tilde{a}\left(\left[p_{\mu_{\nu}}\right] a\right) & =\tilde{a}\left(\sum_{\varrho=\nu}^{\infty}\left[q_{\varrho}\right] a\right)=\tilde{a}\left(\sum_{\varrho=\nu}^{\infty} \frac{1}{\varrho}\left[q_{\varrho}\right] \varrho a\right) \\
& \leqslant \frac{1}{\nu} \tilde{a}\left(\sum_{\varrho=\nu}^{\infty}\left[q_{\varrho}\right] \varrho a\right) \leqslant \frac{1}{v} \tilde{a}\left(b_{0}\right),
\end{aligned}
$$

which yields $\inf _{p \geqslant 1} \tilde{a}\left(\left[p_{p}\right] a\right)=0$. Therefore $\tilde{a}_{F_{0}}$ is continuous, q. e. d.

$R$ is said to be superuniversally continuous, if for any $\left\{a_{\lambda}\right\}_{\lambda \in \Lambda}$ with $a_{\lambda} \leqslant a$ there exists a sequence $\left\{\lambda_{\nu}\right\}_{v=1}^{\infty}, \lambda_{\nu} \in \Lambda$, such that $\bigcup_{\nu=1}^{\infty} a_{\lambda_{\nu}}=\bigcup_{\lambda \in \Lambda} a_{\lambda}$.

Remark 1. A continuous linear functional $\tilde{a}$ is not always universally continuous. On superuniversally continuous space $R$, however, every continuous linear functional on $R$ is obviously universally continuous.

$\left({ }^{13}\right)$ From this it follows that $\tilde{a}\left(a_{\nu}\right) \rightarrow 0$ as $\nu \rightarrow \infty$ for any $a_{\nu} \downarrow_{\nu=1}^{\infty} 0$.

(14) $a^{+}\left(a^{-}\right)$is the positive (resp. negative) part of a, i. e. $a^{+}=a \cup 0$ (resp. $a^{-}=-a \cup 0$ ).
The following lemma was proved by Nakano in the case of modular spaces ([10], Theorem 35.4). As the proof of Lemma 3 is obtained by the quite same way in virtue of (2.2), we omit it here.

LEмпа 3. For any $a \in R$ with $0<\varrho(a)<+\infty$, there exists $\left[p_{0}\right]$ $\left(0 \neq p_{0} \epsilon R\right)$ such that $\varrho\left(\left[p_{0}\right] a\right)=\varrho(a)$ and $\varrho([p] a)=0,[p] \leqslant\left[p_{0}\right]$ implies $p=0 .\left[p_{0}\right] R=\left\{\left[p_{0}\right] x: x \in R\right\}$ is superuniversally continuous as a space.

We denote by $F$ the least normal manifold including $F_{0}$.

LEMIMA 4. If $\tilde{R}$ is total on $R$ and $[p] F$ is superuniversally continuons, then $[p] F$ is semi-regular.

Proof. For any $0<a_{0} \in[p] F$ there exists $a \in F_{0}$ such that $0<a$ $\leqslant a_{0}$. Since $\tilde{R}$ is total, there exists also $0 \leqslant \tilde{a} \in \tilde{R}$ such that $\tilde{a}(a)>0$. Putting $\tilde{a}_{0}(x)=\sup _{0 \leqslant y \leqslant x, y \in F_{0}} \tilde{a}([p] y)$ for any $0 \leqslant x \epsilon F$ and $\tilde{a}_{0}(x)=\tilde{a}_{0}\left(x^{+}\right)$ $-\tilde{a}_{0}\left(x^{-}\right)$for any $x \in \bar{F}$, we obtain a linear functional $\tilde{a}_{0}$ on $[p] F$ and $\tilde{a}_{0}(x)=\tilde{a}(x)$ for all $x \in F_{0}$.

Since $\tilde{a}_{F_{0}}$ is continuous by Lemma 2 and $[p] F$ is superuniversally continuous, $\tilde{a}_{F_{0}}$ is a universally continuous linear functional on $[p] F_{0}$. Hence $\tilde{a}_{0}$ is also such a one on $[p] F$ by the definition of $\tilde{a}_{0}$, because, for any $x \geqslant x_{\lambda} \downarrow{ }_{\lambda \in \Lambda} 0, x, x_{\lambda} \in[p]^{*} F(\lambda \in \Lambda)$ we have

$$
\begin{aligned}
\inf _{\lambda \in A} \tilde{a}_{0}\left(x_{\lambda}\right) & =\inf _{\lambda \in A}\left\{\tilde{a}_{0}(x)-\tilde{a}_{0}\left(x-x_{\lambda}\right)\right\} \\
& =\tilde{a}_{0}(x)-\sup _{\lambda \in A} \tilde{a}_{0}\left(x-x_{\lambda}\right)=\tilde{a}_{0}(x)-\sup _{\lambda \in A}\left\{\sup _{0 \leqslant y \leqslant x-x_{\lambda}, y \in F_{0}} \tilde{a}([p] y)\right. \\
& =\tilde{a}_{0}(x)-\sup _{0 \leqslant y \leqslant x, y \in F_{0}} \tilde{a}([p] y)=\tilde{a}_{0}(x)-\tilde{a}_{0}(x)=0 .
\end{aligned}
$$

As $\tilde{a}_{0}\left(a_{0}\right) \geqslant \tilde{a}(a)>0$ and $\tilde{a}_{0} \in \overline{[p] \bar{F}},[p] F$ is semi-regular, q. e. d. A manifold $M$ of $(R, \varrho)$ is called $\varrho$-singular, if $\varrho(a)=0$ or $=+\infty$ for all $a \in M$. Now we obtain a decomposition theorem:

THEOREM 2.1. Let $(R, \varrho)$ be a quasi-modular space. If $\tilde{R}$ is total on $R$, $R$ can be decomposed into $R=R_{r} \oplus R_{s}$, where $R_{r}$ is semi-regular and $R_{s}$ is $\varrho$-singular.

Proof. Let $R_{r}$ be the totality of all $a \in R$ such that $[a] R$ is semiregular. Then it is clear that $R_{r}$ is a normal manifold of $R$ and $\overline{\left\{R_{r}{ }^{\perp}\right\}}$ $=\{0\}$, because, if $\bar{a}(x) \neq 0$ for some $\bar{a} \in \bar{R}$ and $x \in R_{r} \perp$, then there exists $[p]\left(p \in R_{r}{ }^{\perp}\right)$ such that $[p] R$ is semi-regular ([10], Theorem 24.1). Therefore it suffices to prove that $R_{r}{ }^{\perp}=R_{s}$ is $\varrho$-singular.

Let $0<\varrho(a)<+\infty$ hold for some $0 \leqslant a \in R_{s}$. Then, in virtue of Lemma 4, we may assume without loss of generality that $[a] R$ is superuniversally continuous and $\varrho([p] a)=0$ implies $[p] a=0$. Let $E_{v}(\nu \geqslant 1)$ be the totality of all $[p](p \in R)$ such that $[p] \leqslant[a]$ and $\varrho(v[p] a)<+\infty$. Putting $\left[p_{\nu}\right]=\bigcup_{[p] \in E_{v}}[p]$, we have to consider the following two cases, 
(i) $\left[p_{v}\right]=[a]$ holds for each $v \geqslant 1$; and

(ii) $\left[p_{v_{0}}\right]_{\ddagger}[a]$ holds for some $v_{0} \geqslant 1$.

If (i) holds, then for each $v \geqslant 1$ there exists a mutually orthogonal sequence of projectors: $\left\{\left[p_{v, \mu}\right]\right\}_{\mu=1}^{k_{v}}$ such that $\left[p_{v, \mu}\right] \in E_{y}$, for all $1 \leqslant \mu \leqslant k_{v}$ and

$$
\varrho\left(a-\sum_{\mu=1}^{k_{\nu}}\left[p_{v, \mu}\right] a\right) \leqslant \frac{1}{2^{\nu+1}} \varrho(a)
$$

in virtue of $(2.2)$. We put also $\bigcup_{\mu=1}^{k_{v}}\left[p_{v, \mu}\right]=\left[q_{v}\right]$ for each $v \geqslant 1$ and $\left[p^{\prime}\right]=\bigcup_{\nu=1}^{\infty}\left([a]-\left[q_{v}\right]\right)$. Since

$$
\varrho\left(\left([a]-\left[q_{v}\right]\right) a\right) \leqslant \frac{1}{2^{p+1}} \varrho(a)
$$

holds for each $\nu \geqslant 1$, we have

$$
\varrho\left(\left[p^{\prime}\right] a\right) \leqslant \sum_{\nu=1}^{\infty} \varrho\left(\left([a]-\left[q_{v}\right]\right) a\right) \leqslant \frac{1}{2} \varrho(a) .
$$

Therefore $\varrho\left(\left(1-\left[p^{\prime}\right]\right) a\right) \geqslant \frac{1}{2} \varrho(a)$, hence $\left[p^{\prime \prime}\right]=[a]-\left[p^{\prime}\right] \neq 0$ and $\left[p^{\prime \prime}\right] \leqslant\left[q_{v}\right]$ for all $v \geqslant 1$. Since $\varrho\left(\nu\left[p_{v, \mu}\right] a\right)<+\infty$ for each $\mu$ with $1 \leqslant \mu \leqslant k_{v}$ by the definition of $E_{v}$ and $\left[p^{\prime \prime}\right] \nu a \leqslant \sum_{\mu=1}^{k_{\nu}}\left[p_{v, \mu}\right] \nu a$, we have $\varrho\left(v\left[p^{\prime \prime}\right] a\right)<+\infty$ for each $v \geqslant 1$. This implies $\left[p^{\prime \prime}\right] a \in F_{0}$ and $\left[p^{\prime \prime}\right] F_{0}$ $\left(\subseteq[a] F_{0}\right)$ is semi-regular. From Lemma 1 and Lemma 4 we conclude that $\left[p^{\prime \prime}\right] R$ is also semi-regular. This is a contradiction, because $\left[p^{\prime \prime}\right] R$ $\subseteq[a] R \subseteq R_{r}^{\perp}$

On the other hand, if (ii) holds, we can see that $\left[p_{0}\right] a$ is a strong unit in $\left[p_{0}\right] R$, where $\left[p_{0}\right]=[a]-\left[p_{v_{0}}\right]$ (i. e. for any $x \in\left[p_{0}\right] R$ there exists a real number $\xi_{x} \geqslant 0$ such that $|x| \leqslant \xi_{x}\left[p_{0}\right] a$ holds). Indeed, if such a $\xi_{x}$ does not exist for some $x \in\left[p_{0}\right] R$, then $\left[q_{v}\right]=\left[(|x|-v a)^{+}\right]\left[p_{0}\right] \neq 0$ for all $\nu \geqslant 1$. Now $\left[q_{v}\right]|x| \geqslant \nu\left[q_{\nu}\right] a$ and $\varrho\left(\nu\left[q_{v}\right] a\right)=+\infty$ for all $\nu>v_{0}$ This implies, for any $\mu \geqslant 1$ and for some $\sigma$ with $(\mu+\sigma) / \mu>v_{0}$,

$$
\varrho\left(\frac{1}{\mu}|x|\right) \geqslant \varrho\left(\frac{1}{\mu}\left[q_{\mu+\sigma}\right] x\right) \geqslant \varrho\left(\frac{\mu+\sigma}{\mu}\left[q_{\mu+\sigma}\right] a\right)=+\infty,
$$

which contradicts (p.4) too.

Now we put, for any $0 \leqslant y \epsilon R$,

$$
\begin{aligned}
f_{0}(y) & =\sup _{n \geqslant 1}\left\{\sum_{\nu=1}^{n} \xi_{\nu} \varrho\left(\left[p_{\nu}\right] a\right)\right\} \\
\left(\left[p_{0}\right]\right. & \left.=\left[p_{1}\right]+\ldots+\left[p_{n}\right], \sum_{\nu=1}^{n} \xi_{\nu}\left[p_{,}\right] a \leqslant y\right)
\end{aligned}
$$

and for any $y \in R$

$$
f(y)=f_{0}\left(y^{+}\right)-f_{0}\left(y^{-}\right) .
$$

It is not diffieult to verify that $f$ is a universally continuous linear functional on $R$ and $f\left(\left[p_{0}\right] a\right)=\varrho\left(\left[p_{0}\right] a\right)>0$. This contradicts also $R_{r}^{\perp}=R_{s}$. Thus we have proved that $R_{s}$ must be $\varrho$-singular, q. e.d.

Remark 2. Since a diserete space is semi-regular, $R_{s}$ is alwars non-atomic.

As for the singular part $R_{8}$ we have

THEOREM 2.2. $R_{s}$ can be decomposed into $R_{s}=R_{\infty} \oplus \mathscr{N}$, where $\mathscr{N}$ $=\left\{x: x \in R_{s}, \varrho(\xi x)=0\right.$ for each $\left.\xi\right\}$ and $R_{\infty}$ is the normal manifold $\Re \perp$ $R_{\infty}$ has a strong unit $e$.

Proof. It is clear that $\Re$ is a semi-normal manifold. Let $0 \leqslant a=\bigcup_{\lambda \in A} a_{\lambda}$, where $0 \leqslant a_{\lambda} \epsilon \mathfrak{i}$ for each $\lambda \epsilon \Lambda$. Then, putting $\left[p_{\lambda}\right]=\left[\left(2 a_{\lambda}-a\right)^{+}\right](\lambda \in \Lambda)$, we see that $2\left[p_{\lambda}\right] a_{\lambda} \geqslant\left[p_{\lambda}\right] a$ and $\left[p_{\lambda}\right] \uparrow_{\lambda \epsilon_{\Lambda}}[a]$, hence $0 \leqslant \varrho(a)=\sup _{\lambda \epsilon_{\Lambda}} \varrho\left(\left[p_{\lambda}\right] a\right)$ $\leqslant \sup \left(2\left[p_{\lambda}\right] a_{\lambda}\right)=0$. From this it follows that $\Re$ is a normal manifold.

Thus we obtain obviously that $R_{s}=\Re \oplus R_{\infty}$ because of $\Re^{\perp}=R_{\infty}$

Now we denote by $S$ the set $\left\{a: 0 \leqslant a \epsilon R_{\infty}, \varrho(a)=0\right\}$, and we see clearly that $S$ is directed (with respect to the relation $\leqslant$ ). In the sequel we shall show that $\bigcup_{a \in S} a$ exists.

First we shall prove that for any $[p](p \in R)$ we can find $0 \neq[q]$ $\leqslant[p]$ such that the set $\{[q] a\}_{(a \in S)}$ is order-bounded.

Suppose the contrary case. Then, there exists an element $0 \leqslant p \epsilon R_{\infty}$ such that the set $\left\{\left[p^{\prime}\right] a\right\}_{(a \in S)}$ is not order-bounded for any $0 \neq\left[p^{\prime}\right] \leqslant$ $\leqslant[p]$. Putting $\left[p_{a}^{n}\right]=\left[(|a|-n p)^{+}\right](a \in S, n=1,2, \ldots)$, we have $\varrho\left(n\left[p_{a}^{n}\right] p\right)$ $\leqslant \varrho\left(\left[p_{a}^{n}\right] a\right)=0$ and $\left[p_{a}^{n}\right] \uparrow_{a \in S}[p]$ for all $n \geqslant 1$, because $\cup\left[p_{a}^{n}\right]=\left[p_{0}\right] \neq$ $\lessgtr[p]$ for some $n \geqslant 1$ implies $n\left[p^{\prime}\right] p \geqslant\left[p^{\prime}\right] a$ for all $a \in S$, i. e. the set $\left\{\left[p^{\prime}\right] a\right\}_{(a \in S)}$ is order-bounded, where $\left[p^{\prime}\right]=[p]-\left[p_{0}\right] \neq 0$. Now, since $\varrho(n p)=\sup _{a \in S} \varrho\left(\left[p_{a}^{n}\right] p\right)$ by $(2.2)$, we have

$$
\varrho(n p)=0 \quad(n=1,2, \ldots),
$$

which contradicts that $p \in R_{\infty}$.

Secondly, let the set $\{[q] a\}_{(a \in S)}$ be order-bounded and $b=\bigcup_{a \in S}[q] a$. Since $\left[p_{a}\right]=\left[\left([q] a-\frac{1}{2} b\right)^{+}\right] \uparrow_{a \in S}[q]$ and $\varrho([q] a)=0$ for all $a \epsilon S$, we have $\varrho\left(\frac{1}{2}\left[p_{a}\right] b\right) \leqslant \varrho\left(\left[p_{a}\right] a\right)=0$ and $\varrho\left(\frac{1}{2} b\right)=0$ on account of $(2.2)$.

Now from above, we can find a mutually orthogonal system of elements $\left\{b_{\gamma}\right\}_{(\gamma \in \Gamma)}$ such that $b_{\gamma}=\bigcup_{a \in S}\left[b_{\gamma}\right] a, \varrho\left(\frac{1}{2} b_{\gamma}\right)=0(r \in \Gamma)$ and $\bigcup_{\gamma \in \Gamma}\left[b_{\gamma}\right]$ $=\left[R_{\infty}\right]$. From this it follows that $\bigcup_{\gamma^{\epsilon} \Gamma} \frac{1}{2} b_{\gamma} \in R$, hence $\bigcup_{\gamma \in \Gamma} b_{\gamma}=\bigcup_{\gamma \in \Gamma} \bigcup_{a \in S}^{\gamma^{\epsilon} \Gamma}\left[b_{\gamma}\right] a$ $=\bigcup_{a \in S} \bigcup_{\gamma \in \Gamma}\left[b_{\gamma}\right] a=\bigcup_{a \in S} a=e \epsilon R$. This $e^{\epsilon}$ is a strong unit in $R_{\infty}^{\gamma^{\epsilon} \Gamma}$, because Studia Mathematica $\mathrm{XXII}$ 
$x \in R_{\infty}$ implies $\varrho(\alpha x)=0$ for some $\alpha>0$ and consequently $\alpha|x| \leqslant e$ from the definition of $e$, q. e. d.

Remark 3. It may happen for this $e$ that $\varrho(e)=+\infty$. $\varrho(a)\left(a \in R_{\infty}\right)$ is not, therefore, a (convex) modular on $R_{\infty}$ in general (cf. (iii) in $\S 1$ ).

Now we can remove the additional condition ( $p .5)$ imposed on $\varrho$ and obtain a general result:

THEOREM 2.3. Let $\varrho$ be an arbitrary quasi-modular on $R$ (condition ( .5$)$ is not assumed for $\varrho$ ) and $\tilde{R}$ be total on $R$. Then we have $R=(R, \varrho)$ $=R_{y} \oplus R_{\infty} \oplus \Re$, where $R_{\gamma}, R_{\infty}$ and $\Re$ are the same as in theorems 2.1 and 2.2 :

Proof. Suppose $\varrho^{\prime}$ be a quasi-modular defined by the formula (2.3) and $\varrho$. From theorems 2.1 and 2.2 it follows that $R=\left(R, \varrho^{\prime}\right)=R_{\gamma} \oplus R_{\infty}$ $\oplus \Re$. As $\varrho \leqslant \varrho^{\prime}, \varrho(a)=0$ holds for each $a \epsilon \Re$. Also $\varrho(a)=0$ or $+\infty$ holds for every $a \epsilon R_{\infty}$, since, in the contrary case, we may find $f \in \bar{R}$ such that $f(a)>0$ for some $a \in R_{\infty}$, in the quite same manner as (2.4), q. e.d.

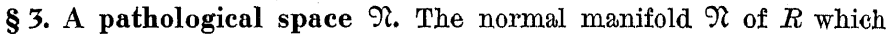
appeared in the previous section is very pathological, if it exists. In fact, $\boldsymbol{N}$ has the following properties:

(3.1) $\Re$ is universally complete $\left({ }^{15}\right)$;

(3.2) $\Re$ is non-atomic;

(3.3) $\tilde{\mathfrak{N}}$ is total on $\mathfrak{N}$ and each $\tilde{a} \epsilon \tilde{\mathfrak{N}}$ is continuous;

(3.4) $\bar{\Re}=\{0\}$.

Indeed, ( $\rho .3$ ) and the fact that $\varrho(a)=0$ for each $a \in \Re$ imply (3.1). (3.2), (3.3) and (3.4) follow from the construction of $\mathfrak{N}$ immediately.

Remark 4. From (3.1) and (3.2) we see clearly that we can define no semi-continuous $\left({ }^{16}\right)$ semi-norm (or quasi-norm) on $\mathfrak{N}$ which is not identically zero.

It is interesting that $\Re$ has the quite similar aspect as discrete spaces in spite of the fact that $\Re$ is non-atomic, as is shown below.

Now let $\mathfrak{F}$ be the proper space of $\mathfrak{N}$, i. e. the topological space of all maximal ideals $\mathfrak{p}\left({ }^{17}\right)$ of projectors $[p]$ on $\Re$ with a neighbourhood system $\left\{U_{[p]}\right\}$ for each $\mathfrak{p} \in \mathfrak{E}$, where $U_{[p]}$ is a set of all $\mathfrak{p}^{\prime} \in \mathfrak{E}$ to which $[p]$ belongs ( $\left.{ }^{18}\right)$.

(15) This means that for any mutually orthogonal system of elements $\left\{a_{\lambda}\right\}_{\lambda \epsilon_{A}}$, there exists $\bigcup_{\lambda \in \Lambda} a_{\lambda} \in R$.

(16) A semi-norm (or a quasi-norm) on $R$ is called semi-continuous, if $\sup _{\lambda \in A}\left\|a_{\lambda}\right\|$ $=\|a\|$ for any $0 \leqslant a_{\lambda} \uparrow_{\lambda \epsilon_{A}} a$.

$\left({ }^{17}\right)$ The set of projectors $\mathfrak{p}$ is called an ideal, if (i) $p=[p],[p] \leqslant[q]$ implies $[q] \in \mathfrak{p}$; (ii) $p=[p],[q]$ implies $p \cdot[p][q]$; (iii) $p p[0]$.

(18) $U_{[p]}$ is both open and compact.
An element $\mathfrak{p}_{0} \in \mathbb{E}$ is called bounded point of $\mathfrak{N}$ [9], if there exists an $a \in \Re$ such that the relative spectrum $\left(^{19}\right)$ of $b$ by $a,\left(b / a, \mathfrak{p}_{0}\right)$, is finite for all $b \in \mathfrak{N}$, and an element $\mathfrak{p} \in \mathbb{F}$ is called transcendental, if for any sequence of neighbourhoods $\left\{U_{\left[p_{v}\right]}\right\}_{(v \geqslant 1)}$ of $\mathfrak{p} \bigcap_{p=1}^{\infty} U_{\left[p_{v}\right]}$ is a neighbourhood of $\mathfrak{p}$ too.

We denote by $C_{\tilde{a}}(\tilde{a} \epsilon \tilde{\mathscr{N}})$ the eharacteristic set of $\tilde{a}$ in $\mathfrak{E}$, i. e. $C_{\tilde{a}}=\left(\bigcup_{\tilde{a}[p]=0} U_{[p]}\right)^{c}$. Since $\mathcal{N}$ is universally complete and each $\tilde{a} \epsilon \tilde{\mathfrak{N}}$ is continuous, we infer that, for any $\tilde{a} \epsilon \tilde{\mathfrak{R}}, C_{\tilde{a}}$ is composed only of a finite number of transcendental points $\mathfrak{p}_{1}, \mathfrak{p}_{2}, \ldots, \mathfrak{p}_{k}$, by applying the quite same argument given in Theorem 3.1 in [11]. On the other hand, if $\tilde{a} \epsilon \tilde{\mathfrak{\imath}}$ and $C_{\tilde{a}}=\left\{\mathfrak{p}_{0}\right\}$, then $\mathfrak{p}_{0}$ is a bounded point of $\mathfrak{N}$ and

$$
\tilde{a}(x)=\left(\frac{x}{a}, \mathfrak{p}_{0}\right) \quad(x \in \Re)
$$

holds for some $a \epsilon \mathfrak{N}$ with $U_{[a]} \mathfrak{p}_{0}$ in virtue of Theorem 1 in [9]. Therefore we obtain (ef. Theorem 3.1 in [11])

THEOREM 3.1. Let $\mathfrak{U}$ be the set $\bigcup_{\tilde{a} \in \tilde{\mathfrak{R}}} C_{\tilde{a}}$. Then we have

(i) $C_{a}$ is composed only of a finite number of elements of $\mathfrak{\xi}$ which are both transcendental and bounded;

(ii) $\mathfrak{U}$ is dense in $\mathfrak{F}$, i. e. if $\mathfrak{p} \notin U_{[p]}$ for all $\mathfrak{p} \in \mathfrak{U}$, then $[p]=0$ holds.

Proof. (i) was already stated above. (ii) is a direct consequence of (3.3) because, in the contrary case, we can find $0 \neq p \epsilon \Re$ such that $U_{[p]} \cap \mathfrak{U}=\varnothing$ and consequently $\tilde{a}(p)=\tilde{a}([p](p))=0$ for all $\tilde{a} \epsilon \tilde{\mathfrak{N}}$, which yields that $\tilde{\mathcal{N}}$ is not total on $\mathfrak{N}$, q. e. $d$.

It is proved by Nakano ([11], Theorem 1.3) that the cardinal number $\left(^{20}\right)$ of any fundamental neighbourhood system of transcendental point $\mathfrak{p}$ must be singular, when $\mathfrak{p}$ is not an isolated point. Therefore, as $\mathfrak{N}$ is non-atomic, we have from Theorem 3.1 obviously

Remark 5. If the cardinal number of $\mathfrak{F}$ is regular, $\mathfrak{N}$ vanishes.

From the theorem above we obtain the next theorem which shows the extreme resemblance of $\mathfrak{N}$ to discrete semi-ordered linear spaces.

THEOREM 3.2. For any $0 \leqslant a, b \in \Re$ there exists a mutually orthogonal system of projectors $\left\{\left[p_{a}\right]\right\}_{a \in(-\infty, \infty)}$ such that

$$
[a] b=\sum_{a \in(-\infty, \infty)} \alpha\left[p_{a}\right] b,
$$

i. e. every spectrum of $b$ by $a(a, b \in \Re)$ is a point spectrum.

$\left({ }^{19}\right)$ For the definition of a relative spectrum see [10], $\S 10$.

${ }^{(20}$ ) A cardinal number $\beta$ is called singular [11], if (i) $\beta>\mathrm{s}_{0}$; (ii) $\beta>\gamma$ implies $\beta>2^{\gamma}$; (iii) for any system of cardinal numbers $\gamma_{\lambda}<\beta(\lambda \in \Lambda)$ with the density $<\beta$ we have $\sum_{\lambda \in A} \gamma_{\lambda}<\beta$. A cardinal number $\gamma$ is called regular, if there is no singular cardinal number $\leqslant \gamma$. 
Proof. Let $0 \lesseqgtr a, b \in \mathfrak{N}$ and $\mathfrak{p}_{0}{ } \mathfrak{U}=\bigcup_{\tilde{a} \in \tilde{\mathfrak{R}}} C_{\tilde{a}}$ with $\left.U_{[a]}\right]^{\ni} \mathfrak{p}_{0}$. As $\mathfrak{p}_{0}$ is a bounded point of $\mathcal{R}$ in virtue of the above theorem, $\left(b / a, \mathfrak{p}_{0}\right)=\lambda_{p_{0}}$ $<+\infty$. Since the set $\mathfrak{L}_{y}=\left\{\mathfrak{p}: \mathfrak{p} \in U_{[a]},\left|(b / a, \mathfrak{p})-\lambda_{\mathfrak{p}_{0}}\right|<1 / v\right\}^{-}$is open and compact for any $v \geqslant 1$, we can find a sequence of projectors $\left\{\left[p_{\nu}\right]\right\}_{(v \geqslant 1)}$ such that

$$
U_{\left[p_{v]}\right]}=\mathcal{L}_{v} \quad(\nu=1,2, \ldots) .
$$

Since $p_{0}$ is transcendental, there exists $0 \neq p_{0} \epsilon \Re$ with $\left[p_{0}\right] \leqslant \bigcap_{v=1}^{\infty}\left[p_{v}\right]$ and $U_{\left[p_{0}\right]}{ }^{\exists} \mathfrak{p}_{0}$ which yields $(b / a, \mathfrak{p})=\lambda_{\mathfrak{p}_{0}}$ for all $\mathfrak{p} \in U_{\left[p_{0}\right]}$, hence $\left[p_{0}\right] b$ $=\lambda_{\mathfrak{p}_{0}}\left[p_{0}\right] a$. Therefore we see that for any $\mathfrak{p} \in U_{[a]} \cap \mathfrak{U}$ there exists a projector $0 \neq[p]=\left[p_{\mathfrak{p}}\right]$ such that $\left[p_{\mathfrak{p}}\right] b=(b / a, \mathfrak{p})\left[p_{\mathfrak{p}}\right] a$ holds.

Now we denote by $D$ the set of all real numbers $\xi$ for which $\xi=$ $(b / a, \mathfrak{p})=\lambda_{\mathfrak{p}}$ for some $\mathfrak{p} \epsilon U_{[a]} \cap \mathfrak{X}$ holds. Then we put, for $\alpha \in(-\infty, \infty)$,

$$
\left[p_{a}\right]=\left\{\begin{array}{ccc}
0, & \text { if } & \alpha \notin D, \\
\bigcup_{\alpha=\lambda_{p}}\left[p_{p}\right], & \text { if } & \alpha \in D .
\end{array}\right.
$$

As $U_{[a]} \cap \mathfrak{U}$ is dense in $U_{[a]},[a] b=\sum_{\alpha \in(-\infty, \infty)} \alpha\left[p_{\alpha}\right] a$ holds, q. e. d.

\$4. The main theorem. In the sequel let $\varrho$ be an arbitrary quasimodular on $R$. By Theorem 2.3 we have

$$
(R, \varrho)=R_{r} \oplus R_{\infty} \oplus \Re,
$$

provided that $\tilde{R}$ is total on $R$

In order to exclude the pathological space $\Re$, we have to impose an additional condition on $\varrho$ as follows:

(p.0) for any $a \in R$, there exists $b \in R$ such that $b \in[a] R$ and $0<\varrho(b)$.

It is clear that under the condition (p.0) $\Re$ does not appear.

Now we obtain

THEOREM 4.1. If $R=(R, \varrho)$ is non-atomio, $\tilde{R}$ is total on $R$ and $\varrho$ satisfies (p.0), then $R$ becomes a quasi-modular space $\left(R, m_{\ell}\right)$ with a perfect (convex) modular $m_{\varrho}$ constructed from $\varrho$.

Proof. Let $R=(R, \varrho)=R_{\gamma} \oplus R_{\infty}$. As $R_{\gamma}$ is semi-regular, we can define a perfect modular $m_{\gamma}$ on $R_{\gamma}$ in virtue of Theorem $A$ in $\S 1$ ([3], Theorem 3.1). Since $R_{\infty}$ has strong unit $e$, we can also define a convex singular modular $m_{\infty}$ such as

Now putting

$$
m_{\infty}(a)=\left\{\begin{array}{cc}
0, & \text { if } \quad|a| \leqslant e ; \\
+\infty, & \text { otherwise. }
\end{array}\right.
$$

$$
m_{\varrho}(a)=m_{\gamma}\left(\left[R_{\gamma}\right] a\right)+m_{\infty}\left(\left[R_{\infty}\right] a\right) \quad(a \in R),
$$

we obtain a (convex) modular on $R$. As $\bar{R}=\bar{R}_{\gamma} \oplus \bar{R}_{\infty}$ and $\bar{R}_{\infty}=\bar{R}_{\gamma}^{\perp}=\{0\}$, we have $\bar{R}=\bar{R}^{m_{\gamma}}=\bar{R}^{m_{e}}$, whence $m_{0}$ is perfect, q.e.d.

Remark 6. $m_{0}$ is always monotone complete on $R_{\infty}$, because, $m_{\ell}(a)$ $=m_{\infty}(a)<+\infty\left(a \in R_{\infty}\right)$ if and only if $|a| \leqslant e$.

Theorem 3.2 and 3.4 in [3] hold valid too, if we replace the condition that $R$ is semi-regular by that $\tilde{R}$ is total on $R$, as $m_{e}$ is monotone complete on $R_{\infty}$ and $\mathfrak{N}$ vanishes in those cases on account of Remark 4 . For instance, we have

THEOREM 4.2. Let $(R, \varrho)$ be a quasi-modular space and $\tilde{R}$ be total on $R$. In order that $R$ be a Banach space with a semi-continuous norm $\|\cdot\|$, it is necessary and sufficient that we can define a monotone complete modular $m_{\varrho}$ on $R$. In this case $\|\cdot\|$-convergence coincides with that of $\|\cdot\| \cdot \|$ : the modular norm by $m_{e}$.

\$ 5. $(R, \varrho)$ with linear topologies. A linear topology $T$ on a semiordered linear space $R$ is called normal, if it contains a fundamental neighbourhood system $\left\{U_{\lambda}\right\}_{\lambda \epsilon_{A}}$ of 0 satisfying the condition: $U_{\lambda}{ } a,|\dot{a}|$ $\geqslant|b|$ implies $b \in U_{\lambda}$ for each $\lambda \in \Lambda$. Also it is called o-compatible if it is normal and contains a fundamental system of neighbourhood of 0 composed of order-closed sets [12]. If $\tilde{R}$ is total on $R$, it is clear that the weak absolute topology $\mathfrak{I}_{S}^{*}(R, \tilde{R})\left({ }^{21}\right)$ induced from $\tilde{R}$ is a locally convex separated linear topology which is normal. Conversely, let $\mathfrak{T}$ be a locally convex separated linear topology on $R$ which is normal. From HahnBanach's Theorem it follows that for any $0 \neq a \epsilon R$ there exists $f \epsilon R^{\prime}$ (the space of all $\mathfrak{T}$-continuous linear functionals on $R$ ) with $f(a)>0$. As $\mathfrak{T}$ is normal and separated, we have $R^{\prime} \subset \tilde{R}$ and $\tilde{R}$ comes to be total on $R$. Thus we obtain, recalling Theorem 4.1,

THEOREM 5.1. Let a quasi-modular space $(R, \varrho)$ be non-atomic. And let $\varrho$ satisfy (p.0) and $\mathfrak{T}$ be a locally convex linear topology on $R$ which is normal and separated. Then we may define a perfect (convex) modular $m_{0}$ on $R$.

The modular $m_{e}$ in Theorem 5.1 may fail to be complete. In order to derive completeness of $m_{e}$, we have to impose some additional conditions on linear topologies on $R$.

A linear topology $\mathfrak{S}$ on $R$ is called monotone complete [12], if $0 \leqslant x_{\lambda} \uparrow_{\lambda \in A}$ and the set: $\left\{x_{\lambda}\right\}_{\lambda \in A}$ is topologically bounded, then $\bigcup_{\lambda \in A} x_{\lambda} \in R$. Since each o-closed convex neighbourhood $V$ of 0 which is also $\mathfrak{T}$-closed determines a semi-continuous semi-norm $\|\cdot\|_{V}$ on $R$, the o-compatible locally convex linear topology $\mathfrak{I}$ is given completely by a system of semi-continuous semi-norms.

(21) The weal absolute topology $\mathcal{I}_{S}^{*}(R, \tilde{R})$ is a linear topology generated by the sets: $\nabla_{\tilde{a}}=\{x:|\tilde{a}||x| \leqslant 1\}(\tilde{a} \epsilon \tilde{R})$ as a fundamental neighbourhood system of 0 . 
THEOREM 5.2. Let $(R, \varrho)$ be a quasi-modular space and a locally convex separated linear topology $\mathfrak{T}$ be o-compatible and monotone complete. Then $(R, \varrho)$ becomes a monotone complete modular space $\left(R, m_{\rho}\right)$ with a (convex) modular $m_{e}$ and $\varrho$-convergence coincides with that of the norm induced by $m_{\varrho}$.

Proof. From Theorem 2.1 and Remark 4 we have $R=R_{\gamma} \oplus R_{\infty}$. Since $\mathfrak{T}$ is monotone complete, $\bar{R}=R_{\gamma}$ holds by virtue of Theorem of [5] stating that every semi-continuous semi-norm is reflexive $\left({ }^{22}\right)$. Hence $m_{e}$ is monotone complete on $R_{\gamma}$ ([10], Theorem 39,5, or [3], Theorem 3.2). On the other hand, $m_{e}$ is a monotone complete modular on $R_{\infty}$, whence $m_{e}$ is also such a one on the whole space $R$. The remainder of this theorem is obtained by the same manner as Theorem 3.2 in [3], q. e. d.

In [4] we proved that $(R, \varrho)$ is decomposed into $R=R_{0} \oplus R_{1}$, where $R_{0}$ is universally complete and $R_{1}$ has a semi-continuous quasi-norm $\|\cdot\|_{0}$ constructed from $\varrho$, and that the necessary and sufficient condition for the completeness of $\|\cdot\|_{0}$ on $R_{1}$ is that $\varrho$ satisfies ([4], Theorem 3.2)

$$
\sup _{x \in R}\left\{\varlimsup_{a \rightarrow 0} \varrho(\alpha x)\right\}<+\infty \text {. }
$$

Since the topology $\mathfrak{I}_{0}$ induced by this quasi-norm $\|\cdot\|_{0}$ is 0 -compatible and monotone complete $\left({ }^{23}\right)$, we have on account of Theorem 5.2

THEOREM 5.3. Let $(R, \varrho)$ be a quasi-modular space which has no infinite dimensional universally complete normal manifold and let $\varrho$ satisfy $\left(\rho .4^{\prime}\right)$. If the quasi-norm $\|\cdot\|_{0}$ of $R$ by $\varrho$ is locally convex, we can define a monotone complete (convex) modular $m_{e}$ on $R$ which induces a norm $\|\mid \cdot\|$ equivalent to $\|\cdot\|_{0}$ (hence $R$ becomes a Banach space in this case).

This theorem is considered as a generalization of Theorem ([6], 2.9) of Mazur and Orlicz on $L_{M}$ and also those of Itô [2], because, in the cases of [6] or [2], we can see without difficulty that the assumptions on $(R, \varrho)$ in Theorem 5.3 are satisfied by the condition setteled on $M$ or $\varrho$ previously.

\section{References}

1] G. Birkhoff, Lattice theory, New York 1948

[2] T. Itô, A generalization of Mazur-Orlicz theorem on function spaces, Jour. Fac. Sci. Univ. Hokkaido Ser. 1, 15, No. 3.4 (1961), p. $221-232$

[3] S. Koshi and T. Shimogaki, On quasi-modular spaces, Studia Math. 21 1961), p. $15-35$.

[4] - On F'norms of quasi-modular spaces, Jour. Fac. Sci. Univ. Hokkaido, Ser. 1, 15, No. 3-4 (1961), p. 202-218.

(22) A semi-norm $\|\cdot\|$ is called reflexive, if $\|x\|=\sup _{-\in \bar{R}} \bar{x}(x)$ for all $x \in R$. 2 and Theorem 3.2 in [4]).
[5] T. Mori, I. Amemiya and $\mathrm{H}$. Nakano, On the reflexivity of semi-continuous norms, Proc. Japan Acad. 31, No. 10 (1955), p. 684-685.

[6] S. Mazur and W. Orlicz, On some elasses of linear metric spaces, Studia Math. 17 (1958), p. 97-119.

[7] J. Musielak and W. Orlicz, On modular spaces, ibidem 18 (1959), p. 49-65. [8] - Some remarks on modular spaces, Bull. Acad. Pol. Sci. 7, No. 11 (1959), p. $661-668$.

[9] H. Nakano, Über ein lineares Funktional auf dem teilweise geordneten Modul, Proc. Imp. Acad. Tokyo 18 (1942), p. 548-552.

[10] - Modulared semi-ordered linear spaces, Tokyo 1950.

[11] - On transcendental points in proper spaces of discrete semi-ordered linear spaces, Jour. Fac. Sci. Univ. Hokkaido, Ser. 1, 12 (1953), p. 105-110.

[12] - Linear topologies on semi-ordered linear spaces, ibidem 12 (1953), p. $87-104$

DEPARTMENT OF MATHEMATICS

DEPARATDO UNIVERSTT, SAPPORO

Reģu par la Rédaction le 16.4.1962 